\title{
SCIENTIFIC REPORTS

\section{Identification of candidate ATP- binding cassette transporter gene family members in Diaphorina citri (Hemiptera: Psyllidae) via adult tissues transcriptome analysis}

\author{
Zhengbing Wang $\mathbb{1}^{1}$, Fajun Tian ${ }^{1}$, Lijun Cai ${ }^{2}$, Jie Zhang ${ }^{2}$, Jiali Liu ${ }^{1} \&$ Xinnian Zeng ${ }^{1 *}$
}

The ATP-binding cassette (ABC) transporters exist in all living organisms and play major roles in various biological functions by transporting a wide variety of substrates across membranes. The functions of $A B C$ transporters in drug resistance have been extensively studied in vertebrates; however, they are rarely characterized in agricultural pests. The Asian citrus psyllid, Diaphorina citri, is one of the most damaging pests of the Citrus genus because of its transmission of Huanglongbing, also known as Yellow Dragon disease. In this study, the next-generation sequencing technique was applied to research the $A B C$ transporters of $D$. citri. Fifty-three $A B C$ transporter genes were found in the RNA-Seq data, and among these $A B C$ transporters, 4, 4, 5, 2, 1, 4, 18 and $15 A B C$ proteins belonged to the $A B C A-A B C H$ subfamilies, respectively. Different expression profiles of 52 genes between imidacloprid-resistant and imidacloprid-susceptible strains were studied by $q R T-P C R ; 5$ ABCGs and $4 A B C H$ s were significantly upregulated in the imidacloprid-resistant strain. In addition, five of the nine upregulated genes were widely expressed in adult tissues in spatial expression analysis. The results suggest that these genes may play key roles in this phenotype. In general, this study contributed to our current understanding of $D$. citri resistance to insecticides.

The ATP-binding cassette $(\mathrm{ABC})$ transporter family is one of the largest families of membrane proteins and universally exists in all living organisms on Earth ${ }^{1}$. The first one was found in prokaryotes. In humans, $48 \mathrm{ABC}$ family members have been identified ${ }^{2}$. The majority of these proteins are membrane-bound primary active transporters that transport various molecules across all cell membranes by binding $\mathrm{ATP}^{3}$. Based on the components of their ATP-binding domain(s), also known as nucleotide-binding domains (NBDs), they are classified as ABC transporters. Each NBD contains three characteristic motifs: ABC signature C, Walker A box and Walker B box. The function of NBDs is to bind and hydrolyse ATP to provide energy for substrate transportation. In addition, the ABC transporters also contain a transmembrane domain (TMD) which usually consists of five to seven membrane helices and participates in the recognition of the substrates. Some eukaryotic ABC transporters are composed of 2 NBDs and 2 TMDs, known as full-transporters, whereas those with only 1 NBD and 1 TMD are called half-transporters. The latter require either homodimers or heterodimers to form a functional unit ${ }^{4,5}$. According to the homology of the NBD sequences, the ABC transporter family is classified into 8 subfamilies, named ABCA-ABCH.

In recent years, $\mathrm{ABC}$ transporters have received increasing attention regarding detoxification. In humans, the overexpression of various ABC transporters in cancer cells can efficiently remove the anticancer drugs from the cells, thus reducing the efficacy of drugs. The development of multidrug resistance (MDR) in cancer cells is one of the major reasons for the failure of cancer chemotherapy ${ }^{6,7}$. In arthropods, ABC transporters are usually associated with insecticide resistance by reducing toxic concentrations in cells/tissues ${ }^{8-10}$. For instance, ABCG4

\footnotetext{
${ }^{1}$ Guangdong Engineering Research Center for Insect Behavior Regulation, Key Laboratory of Bio-Pesticide Innovation and Application, College of Agriculture, South China Agricultural University, Guangzhou, 510642, China. ${ }^{2}$ State Key Laboratory of Ecological Pest Control for Fujian and Taiwan Crops, Institute of Applied Ecology, Fujian Agriculture and Forestry University, Fuzhou, 350002, China. *email: zengxn@scau.edu.cn
} 
correlates with Cry1Ac resistance in Plutella xylostella ${ }^{11}$. Some genes of the ABCB, ABCC, ABCD and ABCG subfamilies are upregulated in strains of Laodelphax striatellus resistant to chlorpyrifos, deltamethrin and imidacloprid $^{12}$. ABC transporters (Mdr50, Mdr65, and Mrp1) are involved in DDT resistance in Drosophila melanogaster ${ }^{13}$. In a study of the interaction between permethrin and verapamil (an inhibitor of ABC transporters) in Anopheles stephensi, the toxicity of permethrin is increased approximately 5 -fold with the inhibition of ABC transporters; concurrently, the expression levels of ABCB6 and ABCG4 are significantly upregulated ${ }^{14}$. It has been reported that $\mathrm{ABC}$ transporters are associated with the transport and/or resistance to 27 different insecticides belonging to 9 distinct chemical classes of insecticides (carbamates, macrocyclic lactones, neonicotinoids, organophosphates, pyrethroids, cyclodienes, benzoylureas, phenylpyrazoles, and DDT) ${ }^{15,16}$.

The Asian citrus psyllid (ACP), Diaphorina citri (Hemiptera: Psyllidae) feeds on citrus flush and transmits Candidatus Liberibacter asiaticus (CLas), a phloem-limited bacterium that infects citrus, that causes Huanglongbing, also known as citrus greening disease or Yellow Dragon disease, a destructive disease of citrus. At present, the application of pesticides is the main way to control the $\mathrm{ACP}^{17,18}$. However, the long-term use of chemical insecticide has led to the development of varying levels of resistance to almost all common insecticides in the populations of $D$. citri in many citrus producing areas ${ }^{19-21}$. The decreased insecticide sensitivity of $D$. citri was related to the increased activities of esterases (ESTs), glutathione S-transferases (GSTs), and cytochrome P450 monooxygenases (P450s) ${ }^{22-24}$. A recent study showed that four P450s, one GST, and one EST of D. citri were associated with imidacloprid resistance ${ }^{25}$.

The development of insecticide resistance seriously impacts the effectiveness of chemical control strategies. Therefore, it is necessary to comprehensively understand the mechanism of insecticide resistance. However, as xenobiotic transporters, the role of the $\mathrm{ABC}$ transporters in insecticide resistance in $D$. citri has not been determined. To identify the ABC transporter genes in D. citri, transcriptome analysis was applied in this study. In total, fifty-three $\mathrm{ABC}$ transporter genes were found, which were classified into eight subfamilies (A-H) by phylogenetic analysis. The detailed sequence comparisons of the eight subfamilies with other species (D. melanogaster, Bombyx mori, Tribolium castaneum, Tetranychus urticae, and Bemisia tabaci) shed light on our understanding of the evolution of the $\mathrm{ABC}$ transporter family among the six species. In addition, the expression profiles of these genes in imidacloprid-susceptible and imidacloprid-resistant strains were analysed by qRT-PCR. Our results provide valuable information on the mechanism of insecticide resistance in D. citri, and will facilitate the elucidation of the functions of these genes in this citrus pest.

\section{Results and Discussion}

Identification of $\mathrm{ABC}$ transporters in D. citri. A total of $53 \mathrm{ABC}$ transporter genes were found in the transcripts of $D$. citri, including 52 unigenes with full-length open reading frame (ORF) sequences and the lengths of these $\mathrm{ABC}$ transporters ranged from 594 to 2413 amino acids (Table 1). In addition, $91 \mathrm{ABC}$ transporter genes or fragments were also found in the genome of D. citri (Accession, GCA_000475195.1), and all these genes or fragments can be matched with those identified from the transcriptome data (Table S2). Therefore, we speculate that the $53 \mathrm{ABC}$ transporters we identified are very close to representing all of the ABC transporters. Then, we aligned the NBDs by the ClustalW program and constructed a neighbor-joining tree. According to the homology of the NBDs, these $53 \mathrm{ABC}$ transporters were grouped into the $8 \mathrm{~A}-\mathrm{H}$ families (Fig. 1). We identified 4, 4, 5, 2, 1, 4, 18 and $15 \mathrm{ABC}$ proteins belonging to the ABCA-H subfamilies, respectively. All genes of subfamilies ABCA and $\mathrm{ABCC}$ were full-transporters; in subfamily $\mathrm{ABCB}$, full-transporters were not identified. The $\mathrm{ABCD}, \mathrm{ABCG}$, and $\mathrm{ABCH}$ subfamilies comprised only half-transporters. However, subfamilies ABCE and ABCF contained only NBDs (Fig. 2). All of the ABC transporter genes of $D$. citri were submitted to GenBank (Table 1).

ABCA subfamily. Four ABCA transporters were identified in D. citri. Three of them were full-transporters, and one was a single TMD-containing ABCA protein (DcABCA2) (Fig. 2). This subfamily includes the largest $\mathrm{ABC}$ transporter, which is encoded by DcABCA1 (2413 amino acids), and in fact, subfamily ABCA transporters are typically the largest among known $\mathrm{ABCs}^{26}$. A phylogenetic tree was constructed to support the member position for the DcABCAs (Supplementary Fig. S1). DcABCA1 clustered with tetur25g01640 and human HsABCA1, 2, 4 and 7. DcABCA2 reveals a high bootstrap support with a group that is formed by six ABCAs of B. tabaci, and then aligned with two ABCAs of T. castaneum, TcABCA-UA and TcABCA-7A, DmCG31731, BmABC004187 and a sister-group that is formed by seven T. urticae ABCAs; these ABCAs form a clade. DcABCA3 is placed in the human ABCA3 clade, which comprises a sister-group of TcABCAs. The clade also contains two BmABCAs (BmABC007217 and BmABC007221). DcABCA4 clustered with three insect ABCAs and then aligned with five human HsABCAs (HsABCA5, HsABCA6, and HsABCA8-10).

In humans, $\mathrm{ABCA}$ transporters play important functions in lipid transport and metabolism ${ }^{2}$. This includes ABCA1, which transports intracellular cholesterol and phosphatide to lipid-poor apolipoprotein A-I (ApoA-I) to form high-density lipoprotein (HDL) ${ }^{27}$. The expression of ABCA1 in the hippocampus is positively associated with the severity of Alzheimer's disease (AD) ${ }^{28}$. However, the role of the arthropod orthologues of these human $\mathrm{ABCAs}$ is currently unclear, but they might be related to lipid transport based on the high conservation of the structure. Injection of dsRNAs of TcABCA-9A and TcABCA-9B, results in approximately $30 \%$ mortality with severe defects in pupae and pharate adults of T. castaneum ${ }^{29}$.

ABCB subfamily. The ABCB subfamily contains both full-transporters and half-transporters. In D. citri, four $\mathrm{ABCB}$ transporters were identified, and all of them are half-transporters that comprise one TMD and one NBD. In the phylogenetic tree (Supplementary Fig. S2), the four DcABCBs were allocated to 4 clades. DcABCB2 was clearly clustered with five $\mathrm{ABCB}$ from other species and formed a clade. This clade contains BmABC005473, TcABCB-6A, DmCG4225, Btaq001304.1, and HsABCB6. DcABCB3, DcABCB4, and DcABCB5 are located in 


\begin{tabular}{|c|c|c|c|c|c|c|c|c|c|}
\hline Subfamily & Name & Accession no. & Length (aa) & Completeness & $\begin{array}{l}\text { Matched protein } \\
\text { (Accession no.) }\end{array}$ & Species & E value & N-Glc & O-Glc \\
\hline \multirow{4}{*}{ A (4) } & DcABCA1 & MH172490 & 2413 & Complete & AIN44098.1 & Laodelphax striatella & 0 & 11 & 8 \\
\hline & DcABCA2 & MH172491 & 1693 & Complete & XP_016657979.1 & Acyrthosiphon pisum & 0 & 6 & 0 \\
\hline & DcABCA3 & MH172492 & 1674 & Complete & XP_022180700.1 & Myzus persicae & 0 & 0 & 0 \\
\hline & DcABCA4 & MH172493 & 2001 & Complete & XP_023723146.1 & Cryptotermes secundus & 0 & 8 & 0 \\
\hline \multirow{4}{*}{ B (4) } & DcABCB2 & MH172495 & 830 & Complete & XP_021938907.1 & Zootermopsis nevadensis & 0 & 0 & 4 \\
\hline & DcABCB3 & MH172496 & 706 & Complete & XP_015606144.1 & Cephus cinctus & 0 & 3 & 0 \\
\hline & DcABCB4 & MH172497 & 688 & Complete & XP_022199620.1 & Nilaparvata lugens & 0 & 1 & 0 \\
\hline & DcABCB5 & MH172498 & 644 & Complete & XP_018904441.1 & Bemisia tabaci & 0 & 1 & 0 \\
\hline \multirow{5}{*}{$\mathrm{C}(5)$} & DcABCC1 & MH172499 & 1523 & Complete & XP_025201032.1 & Melanaphis sacchari & 0 & 5 & 0 \\
\hline & DcABCC2 & MH172500 & 1407 & Complete & XP_021928372.1 & Zootermopsis nevadensis & 0 & 6 & 0 \\
\hline & DcABCC3 & MH172501 & 1373 & Complete & XP_015366071.1 & Diuraphis noxia & 0 & 0 & 0 \\
\hline & DcABCC4 & MH172502 & 1512 & Complete & XP_018902692.1 & Bemisia tabaci & 0 & 4 & 1 \\
\hline & DcABCC5 & MK090470 & 1343 & Complete & XP_018897575.1 & Bemisia tabaci & 0 & 2 & 0 \\
\hline \multirow{2}{*}{$\mathrm{D}(2)$} & DcABCD1 & MH172503 & 712 & Complete & XP_022192008.1 & Nilaparvata lugens & 0 & 0 & 0 \\
\hline & DcABCD2 & MH172504 & 667 & Complete & XP_018911183.1 & Bemisia tabaci & 0 & 2 & 0 \\
\hline E (1) & DcABCE1 & MH172505 & 610 & Complete & XP_023724484.1 & Cryptotermes secundus & 0 & 0 & 0 \\
\hline \multirow{4}{*}{$\mathrm{F}(4)$} & DcABCF1 & MH172506 & 608 & Complete & KZS19906.1 & Daphnia magna & 0 & 2 & 0 \\
\hline & DcABCF2 & MH172507 & 1113 & Complete & XP_018327498.1 & Agrilus planipennis & 0 & 2 & 1 \\
\hline & DcABCF3 & MH172508 & 629 & Complete & XP_025837481.1 & Agrilus planipennis & 0 & 3 & 0 \\
\hline & DcABCF4 & MH172509 & 711 & Complete & XP_018911520.1 & Bemisia tabaci & 0 & 2 & 0 \\
\hline \multirow{18}{*}{ G (18) } & DcABCG1 & MH172510 & 612 & Complete & XP_018915601.1 & Bemisia tabaci & 0 & 2 & 0 \\
\hline & DcABCG2 & MH172511 & 645 & Complete & XP_018915017.1 & Bemisia tabaci & 0 & 0 & 0 \\
\hline & DcABCG3 & MH172512 & 594 & Complete & XP_022181257.1 & Myzus persicae & 0 & 1 & 0 \\
\hline & DcABCG4 & MH172513 & 632 & Complete & XP_018910658.1 & Bemisia tabaci & 0 & 1 & 0 \\
\hline & DcABCG5 & MH172514 & 695 & Complete & XP_018907924.1 & Bemisia tabaci & 0 & 1 & 2 \\
\hline & DcABCG6 & MH172515 & 711 & Complete & XP_018897078.1 & Bemisia tabaci & 0 & 1 & 0 \\
\hline & DcABCG7 & MH172516 & 623 & Complete & XP_018898633.1 & Bemisia tabaci & 0 & 0 & 0 \\
\hline & DcABCG8 & MH172517 & 644 & Complete & XP_018897241.1 & Bemisia tabaci & 0 & 0 & 2 \\
\hline & DcABCG9 & MH172518 & 627 & Complete & XP_018914482.1 & Bemisia tabaci & 0 & 2 & 0 \\
\hline & DcABCG10 & MH172519 & 910 & Complete & XP_018915492.1 & Bemisia tabaci & 0 & 1 & 0 \\
\hline & DcABCG11 & MH172520 & 728 & Complete & XP_024214083.1 & Halyomorpha halys & 0 & 0 & 0 \\
\hline & DcABCG12 & MH172521 & 609 & Complete & XP_018911164.1 & Bemisia tabaci & 0 & 2 & 0 \\
\hline & DcABCG13 & MH172522 & 649 & Complete & XP_018896422.1 & Bemisia tabaci & 0 & 1 & 0 \\
\hline & DcABCG14 & MH172523 & 638 & Complete & XP_018896422.1 & Bemisia tabaci & 7e-149 & 3 & 0 \\
\hline & DcABCG15 & MH172524 & 609 & Complete & XP_014276473.1 & Halyomorpha halys & 0 & 2 & 0 \\
\hline & DcABCG16 & MH172525 & 646 & Complete & XP_014279356.1 & Halyomorpha halys & 0 & 2 & 0 \\
\hline & DcABCG17 & MH172526 & 706 & Complete & XP_018908689.1 & Bemisia tabaci & 0 & 3 & 0 \\
\hline & DcABCG18 & MK090471 & 606 & Complete & XP_025412973.1 & Sipha flava & 0 & 2 & 0 \\
\hline \multirow{15}{*}{$\mathrm{ABCH}(15)$} & DcABCH1 & MH172527 & 686 & Complete & XP_018896133.1 & Bemisia tabaci & $2 e-164$ & 2 & 0 \\
\hline & DcABCH2 & MH172528 & 703 & Complete & XP_001945365.2 & Acyrthosiphon pisum & 0 & 2 & 0 \\
\hline & DcABCH3 & MH172529 & 949 & Complete & XP_021926127.1 & Zootermopsis nevadensis & $1 e-78$ & 7 & 0 \\
\hline & DcABCH4 & MH172530 & 700 & Complete & AKJ85501.1 & Rhopalosiphum padi & 6e-146 & 1 & 0 \\
\hline & DcABCH5 & MH172531 & 769 & Complete & XP_012522429.1 & Monomorium pharaonis & 0 & 4 & 0 \\
\hline & DcABCH6 & MH172532 & 682 & Complete & XP_018916054.1 & Bemisia tabaci & 0 & 1 & 0 \\
\hline & DcABCH7 & MH172533 & 681 & Complete & AKJ85501.1 & Rhopalosiphum padi & $2 \mathrm{e}-175$ & 3 & 0 \\
\hline & DcABCH8 & MH172534 & 689 & Complete & XP_018896133.1 & Bemisia tabaci & 0 & 2 & 0 \\
\hline & DcABCH9 & MH172535 & 764 & Complete & XP_025206654.1 & Melanaphis sacchari & 0 & 2 & 0 \\
\hline & DcABCH10 & MH172536 & 691 & Complete & XP_025208609.1 & Melanaphis sacchari & 0 & 1 & 0 \\
\hline & DcABCH11 & MH172537 & 685 & Complete & XP_022164150.1 & Myzus persicae & 0 & 4 & 0 \\
\hline & DcABCH12 & MH172538 & 677 & Complete & XP_018917681.1 & Bemisia tabaci & $7 e-114$ & 2 & 0 \\
\hline & DcABCH13 & MH172539 & 717 & Complete & XP_012522429.1 & Monomorium pharaonis & $6 e-165$ & 1 & 0 \\
\hline & DcABCH14 & MH172540 & 645 & N-missing & XP_014274344.1 & Halyomorpha halys & $5 e-91$ & 1 & 0 \\
\hline & DcABCH15 & MK090472 & 676 & Complete & XP_025197526.1 & Melanaphis sacchari & $1 e-109$ & 2 & 0 \\
\hline
\end{tabular}

Table 1. Characterisation of $53 \mathrm{ABC}$ transporters in D. citri. aa, amino acid; N-Glc, N-glycosylation sites; O-Glc, O-glycosylation sites. 


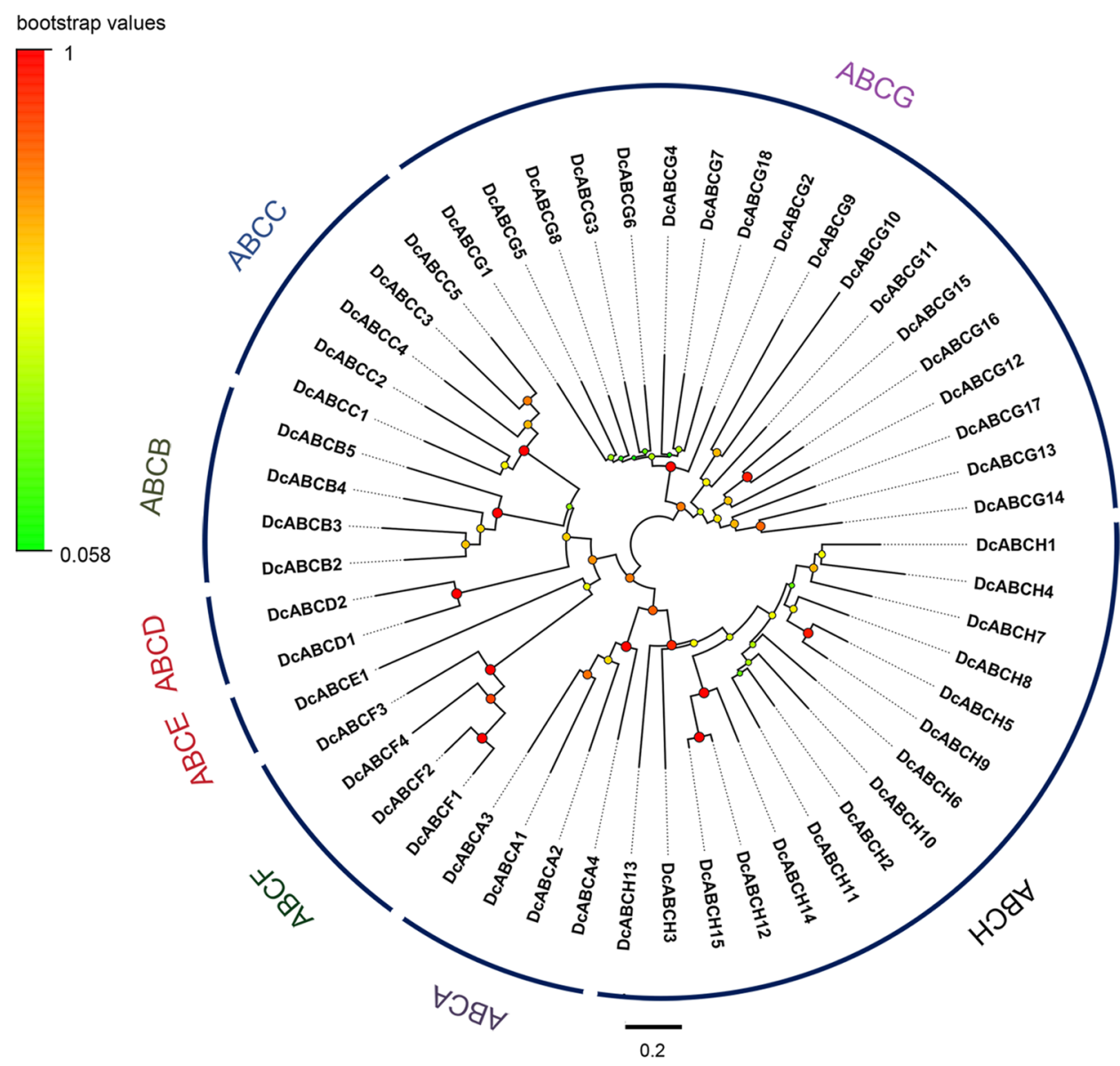

Figure 1. Phylogenetic tree of D. citri $\mathrm{ABC}$ transporters. The amino acid sequences of the nucleotide binding domain (NBD) were used to construct the neighbour-joining tree with the Poisson model. Analysis was performed with MEGA6.0. The bootstrap values resulted from 1000 replications and are displayed in the size and colour of the circles.

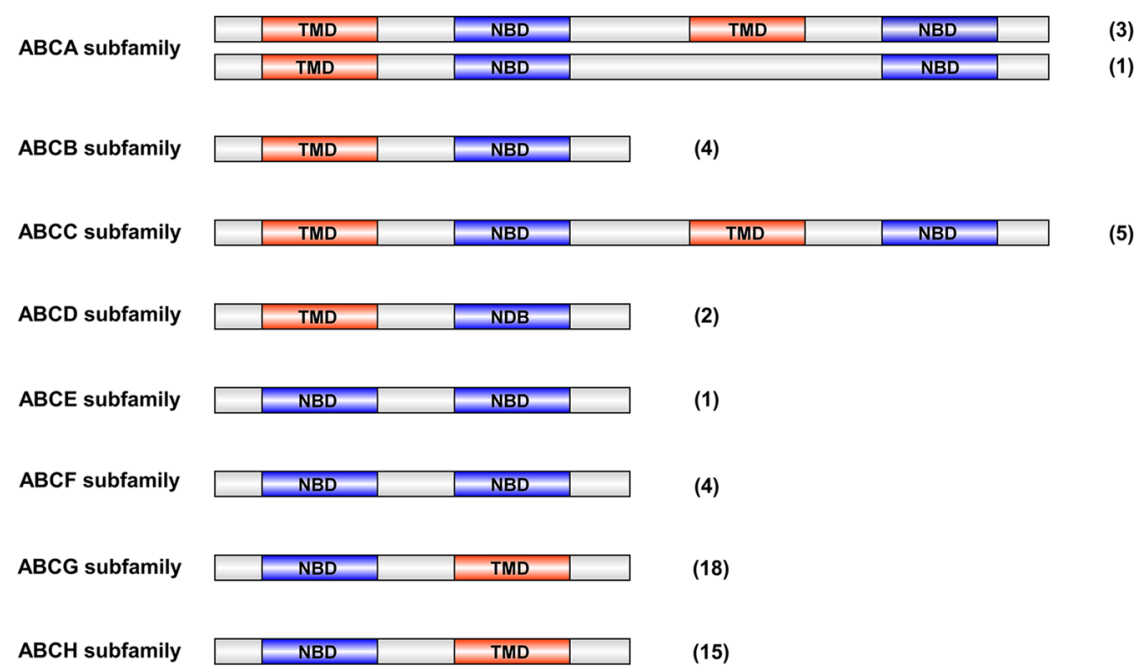

Figure 2. Conserved domain analysis of the $\mathrm{ABC}$ transporters of $D$. citri. The orange stripes indicate the transmembrane domains, TMDs; the blue stripes represent the nucleotide binding domains, NBDs; the numbers in parentheses indicate the number of ABC transporters in each subfamily. 
three other clades similar to DcABCB2. The half transporters in phylogenetic analysis showed obvious orthologous relationships, suggesting that half-transporters have evolutionarily conserved roles in arthropods ${ }^{30}$.

In humans, HsABCB6-8 and HsABCB10 are four mitochondrial ATP-binding cassette transporters. HsABCB6 is associated with multiple cellular functions, including iron homeostasis and porphyrin transport, and is resistant to several cytotoxic agents ${ }^{31}$. HsABCB7 is associated with Refractory anaemia with ring sideroblasts (RARS) ${ }^{32}$. HsABCB8 is involved in protecting the mitochondrial genome through doxorubicin resistance $^{33}$. HsABCB10 is an important player in the protection from oxidative stress ${ }^{34}$. D. melanogaster DmCG4225, a homologous gene of Homo sapiens HsABCB6 was associated with tolerance to cadmium ${ }^{35}$. DmCG3879 (MDR49) is involved in directing germ cell migration through controlling export of a Drosophila germ cell attractant in a signal peptide-independent manner ${ }^{36}$. The homologous gene of HsABCB7 in Aedes aegypti is involved in insecticide resistance ${ }^{37}$.

ABCC subfamily. The $C$ subfamily ABC transporters in humans consist of cystic fibrosis transmembrane conductance regulator (CFTR), membrane-bound sulfonylurea receptors (SURs) and multidrug resistance-associated proteins (MRPs). CFTR (HsABCC7) acts as a chloride channel that is involved in regulating exocrine secretions. SURs (HsABCC8, HsABCC9) binds sulfonylurea and functions as regulators of potassium channels that play a role in modulating insulin secretion. MRPs (HsABCC1-6 and HsABCC10-12) are considered to be important transporters of xenobiotics due to their ability to transport a wide range of substrates (such as drugs, ions, toxins, and endogenous compounds ${ }^{2,38,39}$. Due to their functions, MRPs are the most well characterized in the $\mathrm{ABC}$ transporters subfamily $\mathrm{C}$. All of the human $\mathrm{ABCC}$ transporter genes encode full $\mathrm{ABC}$ transporters; however, both full- and half-transporters were found in insects ${ }^{2,40}$. A human MRP can be classified as a "long" MRP or "short" MRP based on whether it contains a third N-terminal transmembrane domain (TMD0). If it contains a TMD0, it is considered to be a "long" MRP, (HsABCC1-3, HsABCC6, and HsABCC10); on the contrary, the "short" MRPs include HsABCC4, HsABCC5, HsABCC11, and HsABCC12 ${ }^{41}$. In insects, it has been reported that $\mathrm{ABCC}$ is involved in insecticide resistance; for instance, when the nymphs of Nilaparvata lugens are exposed to triazophos, the transcript level of an ABCC shows a significant increase ${ }^{42}$. In Pediculus humanus, silencing PhABCC4 by RNAi leads to an increased susceptibility to ivermectin ${ }^{43}$.

In D. citri, five ABCC transporter genes were identified; all genes contained full-length ORFs and encoded full ABC transporters (Table 1, Fig. 2). In phylogenetic analysis (Supplementary Fig. S3), DcABCC1 clustered with Btabq019529.2 and Btabq000311.1, DmCG6214, two B. mori ABCCs, four human MRPs (HsABCC1, HsABCC2, HsABCC3, and HsABCC6), twenty-three T. urticae ABCCs, and T. castaneum TcABCC-9A. As an orthologue to human MRPs, DmCG6214 is an ATP-dependent, vanadate-sensitive organic anion transporter and transports developmentally significant hormones, such as ecdysteroid and juvenile hormone ${ }^{41}$. DcABCC2 cluster with three human MRPs (HsABCC5, HsABCC11 and HsABCC12), where HsABCC5 and HsABCC11 act as nucleoside transporters; however, the function of HsABCC12 is unknown ${ }^{2,44}$. DcABCC3 and DcABCC5 were placed in a large clade containing HsABCC4, a large cluster of T. urticae ABCCs, seven B. mori ABCCs, a cluster of T. castaneum ABCC5s, and ten D. melanogaster ABCCs. HsABCC4 has the ability to transport a wide variety of endogenous and xenobiotic organic anionic compounds out of the cell; these substrates also include molecules involved in cellular signaling ${ }^{2}$. DcABCC4 clustered with human HsABCC10, DmCG7806, BmABC010636, Tetur03g07840, Btabq004618.1, and TcABCC-4A, and this clade showed clear orthologous relationships. HsABCC10 is known as a drug-efflux pump because it is involved in the transport of amphipathic anions, leading to resistance to a variety of anticancer drugs ${ }^{45}$. In the transcriptomes of $D$. citri, the orthologues of CFTR and SUR are not identified.

ABCD subfamily. The ABCD subfamily transporters are half-transporters in animals with one TMD and one NBD and play a role in transporting fatty acids and acyl-CoA in peroxisomes ${ }^{46}$. Two ABCD transporter transcripts were identified in the transcriptomes of $D$. citri, both of which have full-length ORFs. The same number of $\mathrm{ABCDs}$ was also found in the genome of most other insects ${ }^{47}$ (Table 2). In the phylogenetic tree (Supplementary Fig. S4), D. citri DcABCD1 clustered with B. tabaci Btabq026746.1, T. castaneum TcABCD-6A, D. melanogaster DmCG2316, B. mori BmABC004616, T. urticae tetur05g06640, H. sapiens HsABCD1 and HsABCD2. DcABCD2 is located in the HsABCD3 clade. The phylogenetic analysis reflected clear orthologous relationships with ABCD transporter proteins among these species, suggesting that ABCD transporters are highly conserved in animals.

$A B C E$ and $A B C F$ subfamilies. The $A B C E$ and $A B C F$ subfamilies are quite distinct from other $A B C$ transporters because they only contain two linked NBDs and lack TMDs (Fig. 2). In view of the special structure, $\mathrm{ABCE}$ and $\mathrm{ABCF}$ proteins are involved in biological processes other than transportation. RNAi against Caenorhabditis elegans ABCE, which is also known as an RNase L inhibitor (RLI) in eukaryotes, resulted in embryonic lethality and slow growth, suggesting that $\mathrm{ABCE}$ plays a role in the regulation of translation and transcription $^{48}$. In humans, HsABCE1 has an important role in HIV-1 assembly ${ }^{49}$, and HsABCF1 (ABC50) is associated with promoting translation initiation ${ }^{50}$. In insects, injection of dsRNA specific for T. castaneum TcABCE-3A and TcABCF-2A, led to $100 \%$ mortality in the larvae of T. castaneum $^{29}$.

One $\mathrm{ABCE}$ and four $\mathrm{ABCF}$ transporter genes were identified in the D. citri transcriptomes. Most eukaryotes only have one ABCE and three ABCF genes (Table 2). In the phylogenetic tree (Supplementary Fig. S5), DcABCE1 showed the highest homology with BmABC010129 and TcABCE-3A. ABCFs clustered into well-supported separate clades, with DcABCF1 and DcABCF2 located in the HsABCF1 clade, and DcABCF3 and DcABCF4 positioned at the HsABCF2 and HsABCF3 clades separately. Phylogenetic analysis revealed that the ABCE and ABCF subfamilies were highly conserved. 


\begin{tabular}{|l|l|l|l|l|l|l|l|l|l|}
\hline Species & ABCA & ABCB & ABCC & ABCD & ABCE & ABCF & ABCG & ABCH & TOTAL \\
\hline Homo sapiens & 12 & 11 & 12 & 4 & 1 & 3 & 5 & 0 & 48 \\
\hline Daphnia pulex & 3 & 6 & 24 & 3 & 1 & 3 & 26 & 11 & 82 \\
\hline Tetranychus urticae & 9 & 4 & 39 & 2 & 1 & 3 & 23 & 22 & 103 \\
\hline Tigriopus japonicus & 5 & 5 & 17 & 3 & 1 & 3 & 7 & 5 & 46 \\
\hline Brachionus koreanus & 11 & 19 & 15 & 3 & 1 & 3 & 8 & 2 & 61 \\
\hline Drosophila melanogaster & 10 & 8 & 14 & 2 & 1 & 3 & 15 & 3 & 56 \\
\hline Anopheles gambiae & 9 & 5 & 13 & 2 & 1 & 3 & 16 & 3 & 52 \\
\hline Bombyx mori & 6 & 8 & 15 & 2 & 1 & 3 & 13 & 3 & 51 \\
\hline Helicoverpa armigera & 7 & 11 & 11 & 2 & 1 & 3 & 16 & 3 & 54 \\
\hline Plutella xylostella & 15 & 14 & 21 & 3 & 1 & 3 & 19 & 6 & 82 \\
\hline Tribolium castaneum & 10 & 6 & 35 & 2 & 1 & 3 & 13 & 3 & 73 \\
\hline Apis mellifera & 3 & 7 & 9 & 2 & 1 & 3 & 15 & 3 & 43 \\
\hline Bemisia tabaci & 8 & 3 & 6 & 2 & 1 & 3 & 23 & 9 & 55 \\
\hline Lygus hesperus & 11 & 6 & 12 & 2 & 1 & 3 & 19 & 11 & 65 \\
\hline Diaphorina citri & 4 & 4 & 5 & 2 & 1 & 4 & 18 & 15 & 53 \\
\hline
\end{tabular}

Table 2. Number of each ABC transporter subfamily in different species.

ABCG subfamily. The ABCG transporter family is present in most metazoan species, fungi and plants. Based on the research of predecessors, ABCG half-transporters were only identified in metazoan species except one ABCG gene in P. xylostella (Px007949) ${ }^{51}$. However, full-transporters are widely present in fungi and plant $^{52,53}$. The half-transporters have a reverse domain structure with an NBD at the N-terminus and a TMD at the C-terminus (NBD-TMD), while a functional transporter must be dimeric ${ }^{15}$. In humans, five ABCG transporter family genes have been identified. Among these HsABCGs, four HsABCGs except HsABCG2 were mainly involved in the transportation of dietary lipids, while HsABCG2 (breast cancer resistance protein, BCRP) has a series of substrates, including anticancer drugs, and acts as an $\mathrm{MRP}^{54}$. Among invertebrates, D. melanogaster ABCG members were first characterized, including brown, scarlet, and white genes ${ }^{55}$.

Eighteen ABCG transporter family transcripts were identified in the transcriptomes of D. citri and represent the largest $\mathrm{ABC}$ subfamily in $D$. citri, all of which possess full-length coding sequences and are in accord with half-transporters with the topology TMD-NBD. In the phylogenetic tree (Fig. 3), eight D. citri ABCG genes (DcABCG1-3, DcABCG5-8, and DcABCG18) clustered with potential orthologues of HsABCG1 and HsABCG4 in ABCG clades, where HsABCG1 is involved in regulating the output of cholesterol, while the function of HsABCG4 was not clear ${ }^{56}$. In humans, HsABCG5 and HsABCG8 form a functional heterodimer and play a role in removing plant sterols from the body ${ }^{56}$. In the phylogenetic tree, DcABCG9 and DcABCG10 were two orthologous genes of HsABCG5 and HsABCG8, and all the arthropod orthologues of HsABCG5/HsABCG8 showed a head-to-head arrangement, indicating that DcABCG9 and DcABCG10 may have similar functions as HsABCG5/ HsABCG8. Six genes (DcABCG12-17) clustered with D. melanogaster white, scarlet, and brown and the orthologues of the other species. In D. melanogaster, white, scarlet, and brown are the best-characterized ABCG genes of arthropods, and scarlet or brown takes part in transporting pigment precursors in the Malpighian tubules and relates to the formation of compound eye colour ${ }^{57,58}$. D. melanogaster white mutants show a white-eye colour phenotype, and this phenomenon has also been confirmed in T. castaneum and B. mori ${ }^{29,59}$. However, white is also involved in resistance to pesticide, and downregulation of the white orthologues leads to increased Bt resistance in P. xylostella ${ }^{60}$. In D. citri, DcABCG17 is orthologous to white, DcABCG13 and DcABCG14 are orthologous to scarlet, and three genes (DcABCG12, DcABCG15 and DcABCG16) are orthologous to brown. DcABCG11 clustered with B. tabaci Btabq023890.1, T. castaneum TcABCG-8A, D. melanogaster DmCG3327, and T. urticae tetur17g02510. In D. melanogaster, DmCG3327 (also named E23) is capable of modulating the 20E response ${ }^{61}$, and a similar function has also been found in T. castaneum TcABCG-8A ${ }^{29}$. DcABCG6 clustered with T. castaneum TcABCG-4C, D. melanogaster DmCG3164, B. mori BmABC005202 and two orthologues of B. tabaci. In T. castaneum, TcABCG-4C-dsRNA injected larvae exhibited a rough cuticle as a consequence of desiccation and shrinkage and rapidly caused death during the quiescent stage, in addition, injection of TcABCG-4C-dsRNA into pre-pupae resulted in death at the pupal stage before the pupal-adult molt, while DmCG3164 performs a similar function in Drosophila ${ }^{29,62}$.

ABCH subfamily. The $\mathrm{ABCH}$ transporter family proteins are half-transporters and share the same NBD-TMD topological structure as the $\mathrm{ABCH}$ family. $\mathrm{ABCH}$ transporters were first identified in D. melanogaster and it was then reported that the $\mathrm{ABCH}$ subfamily was only found in arthropods, zebrafish Danio rerio and marine medaka Oryzias melastigma ${ }^{15,63}$. At present, the $\mathrm{ABCH}$ subfamily has not been identified in other species such as mammals, plants and fungi ${ }^{2,52,64}$. We identified fifteen DcABCH genes in the transcriptomes of $D$. citri, fourteen of them have the full-length ORF. In most insect species, only three ABCH members were found, including D. melanogaster, B. mori and T. castaneum which has an excellent genome sequence, however, a large number of $\mathrm{ABCH}$ members were found in three Hemiptera insects (B. tabaci, Lygus Hesperus and D. citri) and two species of arthropods (D. pulex and T. urticae) (Table 2). In the phylogenetic tree (Fig. 4), six DcABCH sequences (DcABCH1, DcABCH4, DcABCH7, DcABCH12 and DcABCH14-15) formed a separate clade, which was similar to the $\mathrm{ABCH}$ of T. urticae $e^{30}$ and D. pulex ${ }^{65}$, suggesting that the diversity of $\mathrm{ABCH}$ proteins in D. citri 


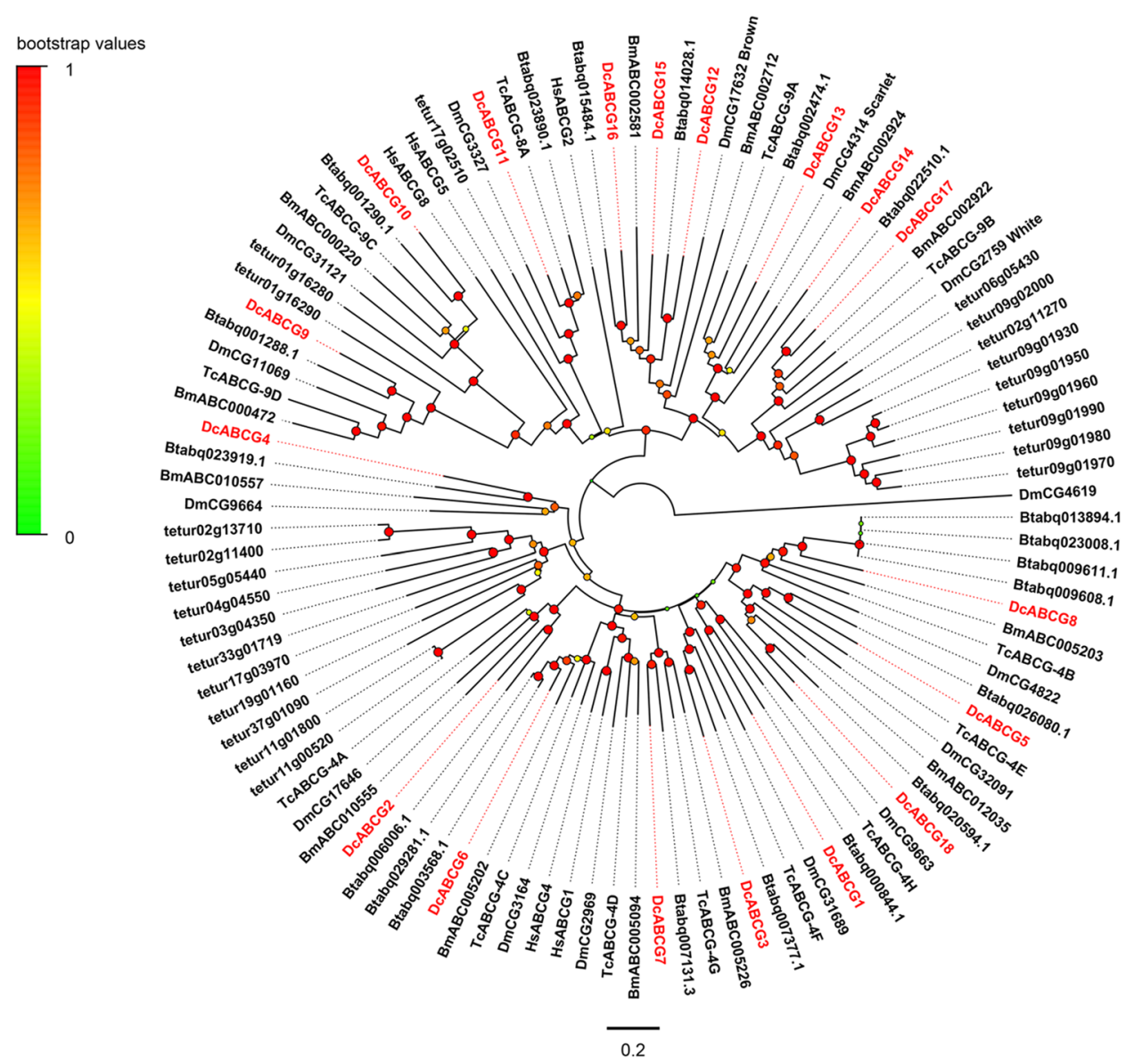

Figure 3. Phylogenetic analysis of ABCG transporters of D. citri and other species. Dm, Drosophila melanogaster; Bm, Bombyx mori; Tc, Tribolium castaneum; tetur, Tetranychus urticae; Btabq, Bemisia tabaci Q; Dc, D. citri (red). The neighbour-joining tree was constructed using MEGA6.0 software and with the Poisson model. The bootstrap values resulted from 1000 replications and are displayed in the size and colour of the circles.

has been due to lineage-specific duplications events, this similar expansion was also found in two Hemiptera insects B. tabaci and L. hesperus ${ }^{26,64}$.

$\mathrm{ABCH}$ plays an important role in insect physiology. In recent years, researchers have been exploring and have a considerable understanding of their physiological function. In Helicoverpa armigera and Manduca sexta, when the larvae were fed with secondary metabolites, the expression of $\mathrm{ABCH}$ subfamily was induced to increase ${ }^{66,67}$. In D. melanogaster, cold hardening treatment resulted in a 2-fold increase in the expression level of DmCG33970 ${ }^{68}$, both DmCG9990 null mutants and RNAi-mediated knockdown DmCG9990 are lethal ${ }^{62,69}$. In addition, DmCG9990 was also found to be associated with the formation of epidermal barrier ${ }^{70}$. In T. castaneum, injection of dsRNA specific for TcABCH-9C, the ortholog of DmCG9990, resulted in desiccation and $100 \%$ larval mortality and a significant reduction in fertility and the egg hatchability. Furthermore, TcABCH-9C dsRNA treated larvae showed a lack of lipids in the epicuticle, and based on these results, the authors inferred that TcABCH-9C was involved in transport of lipids to epicuticle and promoting the formation of waterproof barrier in epicuticle ${ }^{29}$. In a recent study, the ortholog of DmCG9990 in Locusta migratoria, LmABCH-9C, were borne out to be associated with transport of lipids to epicuticle and cuticle barrier formation in epicuticle ${ }^{71}$. In D. citri, DcABCH5 and DcABCH9 are orthologues of DmCG9990 and DmCG33970, respectively (Fig. 4).

Expression profile of DCABCs. The spatial expression profiles of these $A B C$ transporter genes were estimated by analysing the FPKM values. Ten genes (DcABCB5, DcABCD1, DcABCE1, DcABCF2-3, DcABCG6-8, DcABCG10, and DcABCH5) are widely expressed in adult tissues of D. citri. Seven $A B C$ transporter genes (DcABCA1, DcABCA3-4, DcABCC1, DcABCC3-4, DcABCG15, and DcABCH2) showed high expression levels in the abdomen of adults (Fig. 5). To understand the possible role of DcABCs in the insecticide resistance of $D$. citri, qRT-PCR was used to compare the expression of these genes between the imidacloprid-susceptible and imidacloprid-resistant strains. The expression level of DcABCG11 in susceptible strains was too low to be detected; therefore the expression levels of 52 genes were presented. Nine DcABCs were significantly upregulated in the imidacloprid-resistant strains compared to the susceptible strains (Fig. 6), and the upregulated genes were mainly concentrated in the ABCG and ABCH subfamilies. DcABCH4 was upregulated 3.9-fold in 


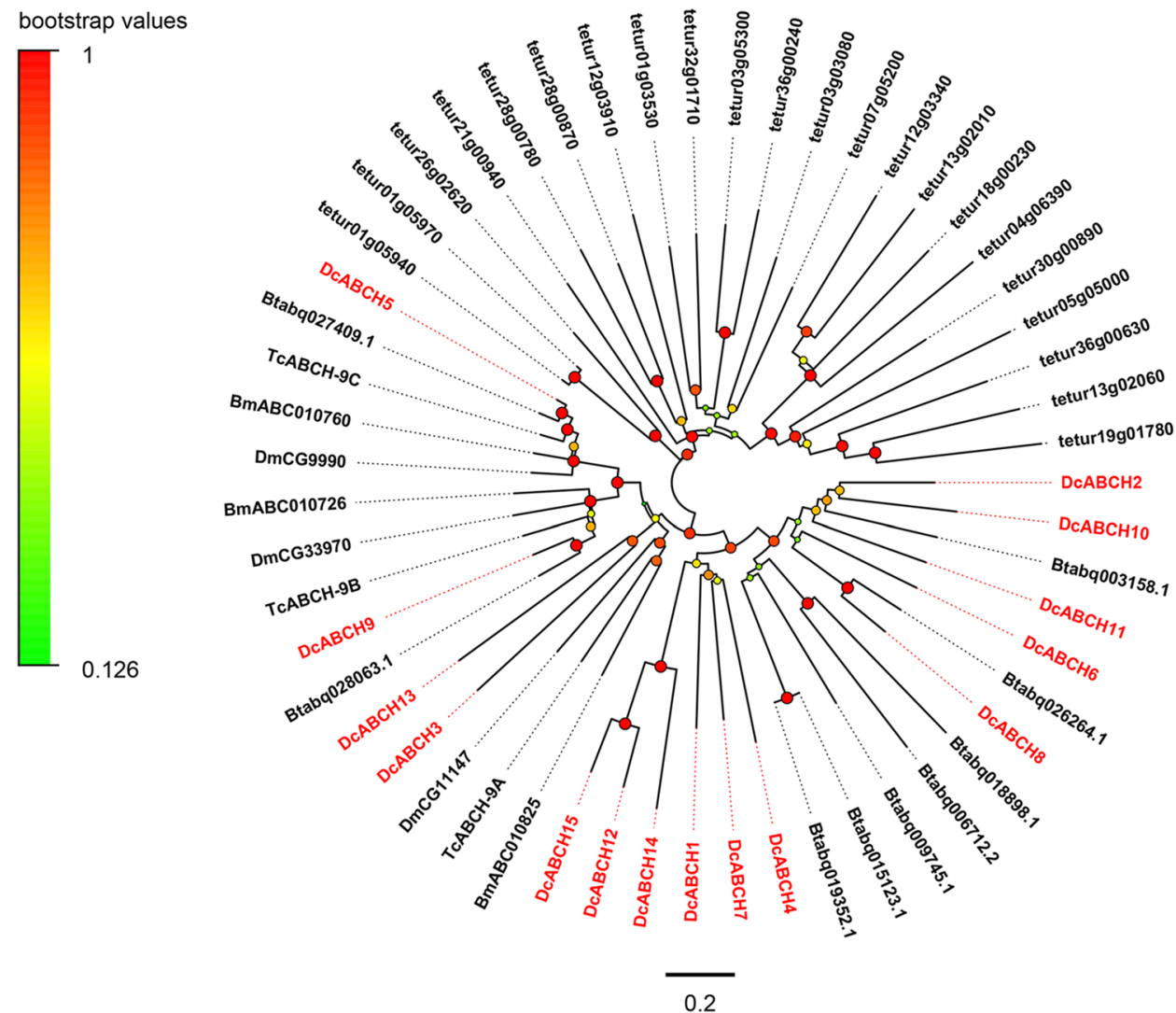

Figure 4. Phylogenetic analysis of $\mathrm{ABCH}$ transporters of $D$. citri and other species. The abbreviations and colour settings are consistent with Fig. 3. The neighbour-joining tree was constructed using MEGA6.0 software and with the Poisson model. The bootstrap values resulted from 1000 replications and are displayed in the size and colour of the circles.

resistant strains, and DcABCG3 and DcABCG9 were upregulated 2.6-fold and 2.9-fold. DcABCG6, DcABCG7, DcABCG10, DcABCH5, DcABCH9, and DcABCH12 were upregulated 1.4-fold to 1.8-fold. Twenty-seven of the $52 \mathrm{DcABCs}$ were down-regulated in resistant strains, and the expression of the remaining 16 DcABCs did not show a significant difference between susceptible and resistant strains.

In arthropods, $\mathrm{ABCB}, \mathrm{ABCC}$ and $\mathrm{ABCG}$ were the three most reported subfamilies associated with insecticide transport or resistance, and the index of insecticide transport or resistance is mainly based on their expression level ${ }^{15}$. In $D$. citri, five ABCG transporters were significantly upregulated in imidacloprid-resistant strains, and similar upregulation of expression has also been reported in other insects. For instance, one ABCG transporter was upregulated in the imidacloprid-susceptible Leptinotarsa decemlineata ${ }^{72}$. Four ABCG transporters were up-regulated in imidacloprid-treated $B$. tabaci ${ }^{64}$. In addition to the ABCG transporter, four ABCH transporters were significantly upregulated in imidacloprid-resistant strains. In the green peach aphid, Myzus persicae, an $\mathrm{ABCH}$ was upregulated under the stress of pirimicarb ${ }^{73}$, suggesting that $\mathrm{ABCH}$ may also be involved in insecticide resistance in insects. The results indicated a potential implication of these genes in imidacloprid resistance. In addition, five of the nine upregulated genes (DcABCG6-8, DcABCG10, and DcABCH5) were expressed widely in adult tissues, which may demonstrate that the wide expression of ABC transporters may contribute to the transport of exogenous substances such as pesticides. Twenty-seven DcABCs were downregulated in resistant strains. Similarly in P. xylostella, Px006766 (PxABCF3) and Px013659 (PxABCA12) were downregulated in chlorpyrifos-resistant and fipronil-resistant strains ${ }^{51}$. This may indicate that not all ABCs are involved in detoxification and may be a physiological adaptation to long-term pesticide pressure.

\section{Conclusions}

The major objective of this study was to identify the ATP-binding cassette transporter gene family in D. citri. In this study, fifty-three genes encoding $\mathrm{ABC}$ transporters were identified in D. citri using RNA-Seq and transcriptomic analysis. Among 8 subfamilies, ABCG and ABCH have more members in D. citri. Moreover, nine genes of these two subfamilies were upregulated in the imidacloprid-resistant strain of adult $D$. citri and five of them were expressed extensively in adult tissues. These results enrich the research content regarding the insecticide resistance mechanism in D. citri and will further facilitate our understanding of imidacloprid-resistance mechanisms in this pest. 


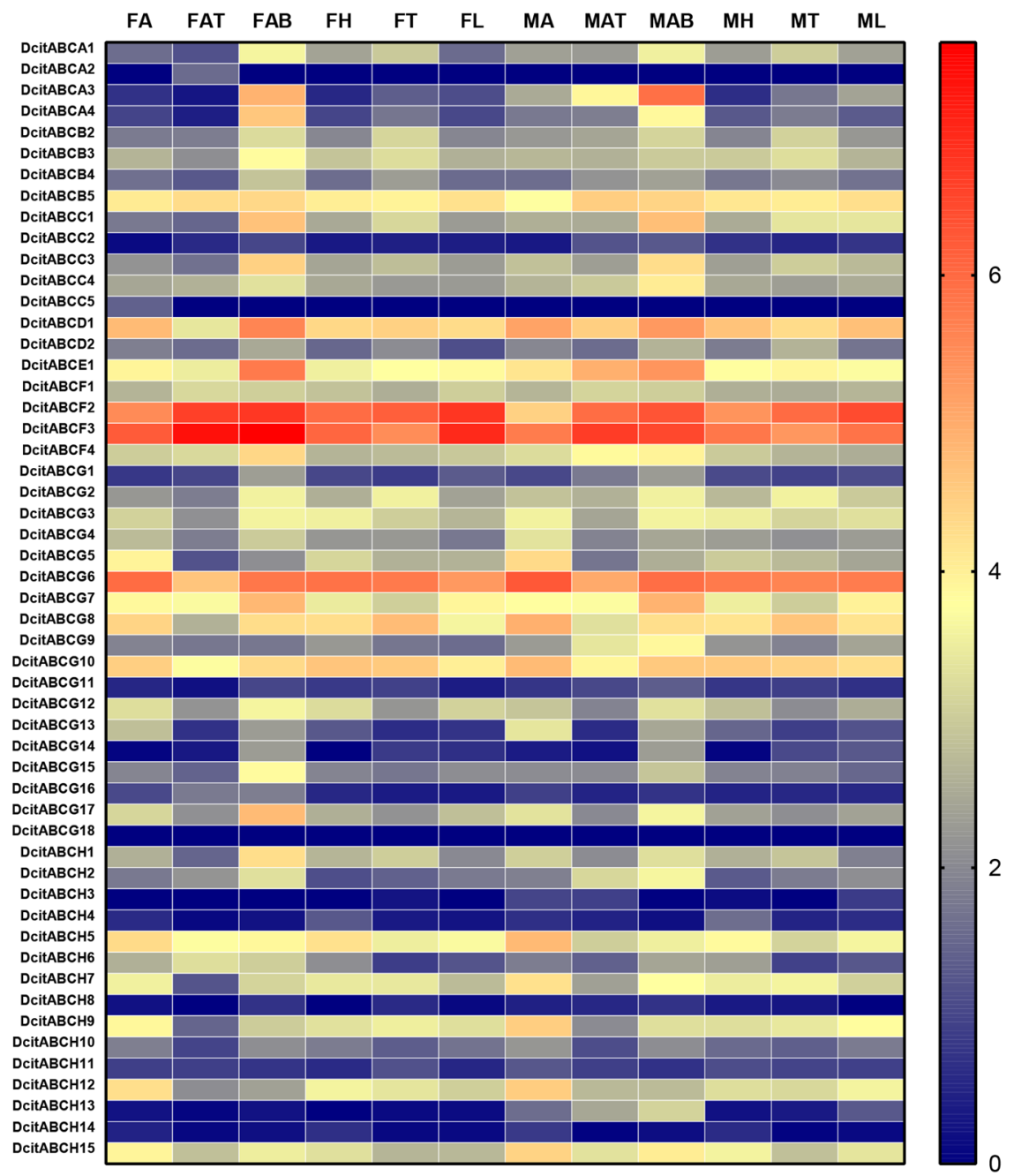

Figure 5. Tissue-specific expression profiles of $A B C$ transporter genes in various tissues of $D$. citri based on FPKM values. The mRNA levels, as represented by $\log 2(\mathrm{FPKM}+1)$ values, are shown in the heat map with colors ranging from blue (low expression) to red (high expression). MA, male antenna; $\mathrm{MH}$, male head; MT, male thorax; ML, male leg; MAB, male abdomen; MAT, male abdomen terminal; FA, female antenna; FH, female head; FT, female thorax; FL, female leg; FAB, female abdomen; FAT, female abdomen terminal.

\section{Materials and Methods}

Insect rearing and strains. Two strains of D. citri were used in this study: laboratory-susceptible strains and imidacloprid-resistant strains. The laboratory susceptible-strains were collected from Murraya exotica on the campus of South China Agricultural University, Guangzhou, Guangdong Province, China, in 2013, and this population was reared in the greenhouse without exposure to any insecticides. The imidacloprid-resistant strains originate from field populations in Guangzhou, Guangdong Province, China, in 2016, and then they were continuously exposed to imidacloprid to select the resistant strains. A 52.19-fold imidacloprid-resistant strain was obtained through 9 generations of continuous selection via the leaf dip method ${ }^{25}$. Both strains were kept rearing on M. exotica in a climate chamber $\left(26^{\circ} \mathrm{C}, 80 \% \mathrm{RH}\right)$ and a $14: 10 \mathrm{~h}$ (light:dark) photoperiod.

Sample collection and RNA Seq. The tissues of insects were dissected from 3-day-old adults of laboratory-susceptible strains. A total of 2000 antennas (including a modicum of tissues of heads), 200 heads (antennas removed), 150 thoraxes, 300 legs, 150 abdomens, and 1000 terminal abdomens (cut from the 5th abdominal segments) were collected from male adults, and the tissues from famale adults had equal numbers. Total RNA from each sample was extracted using TRIzol Reagent (Invitrogen, Waltham, MA, USA). Total RNA samples were quantified and assessed for quality by a NanoDrop 2000 (Thermo Fisher Scientific, Waltham, MA, USA). Transcriptome sequencing was performed on an Illumina HiSeq. 2500 platform (Genewiz, Suzhou, China), and a total of $143.37 \mathrm{~Gb}$ of raw data was acquired. After removing low-quality, adaptor and contaminating sequence reads, $137.22 \mathrm{~Gb}$ of clean reads was obtained. The clean data were assembled by Trinity, and 297,614 unigenes larger than $200 \mathrm{bp}$ were obtained, the unigenes were submitted to InsectBase (http://www. insect-genome.com/data/Diaphorina_citri.transcript.fa.tar.gz $)^{74-76}$. The raw data of the transcriptomes were submitted to the NCBI Short Read Archive (SRA) database (Submission ID: SRP139008) (https://www.ncbi.nlm.nih. gov/sra/SRP139008) ${ }^{76}$. 

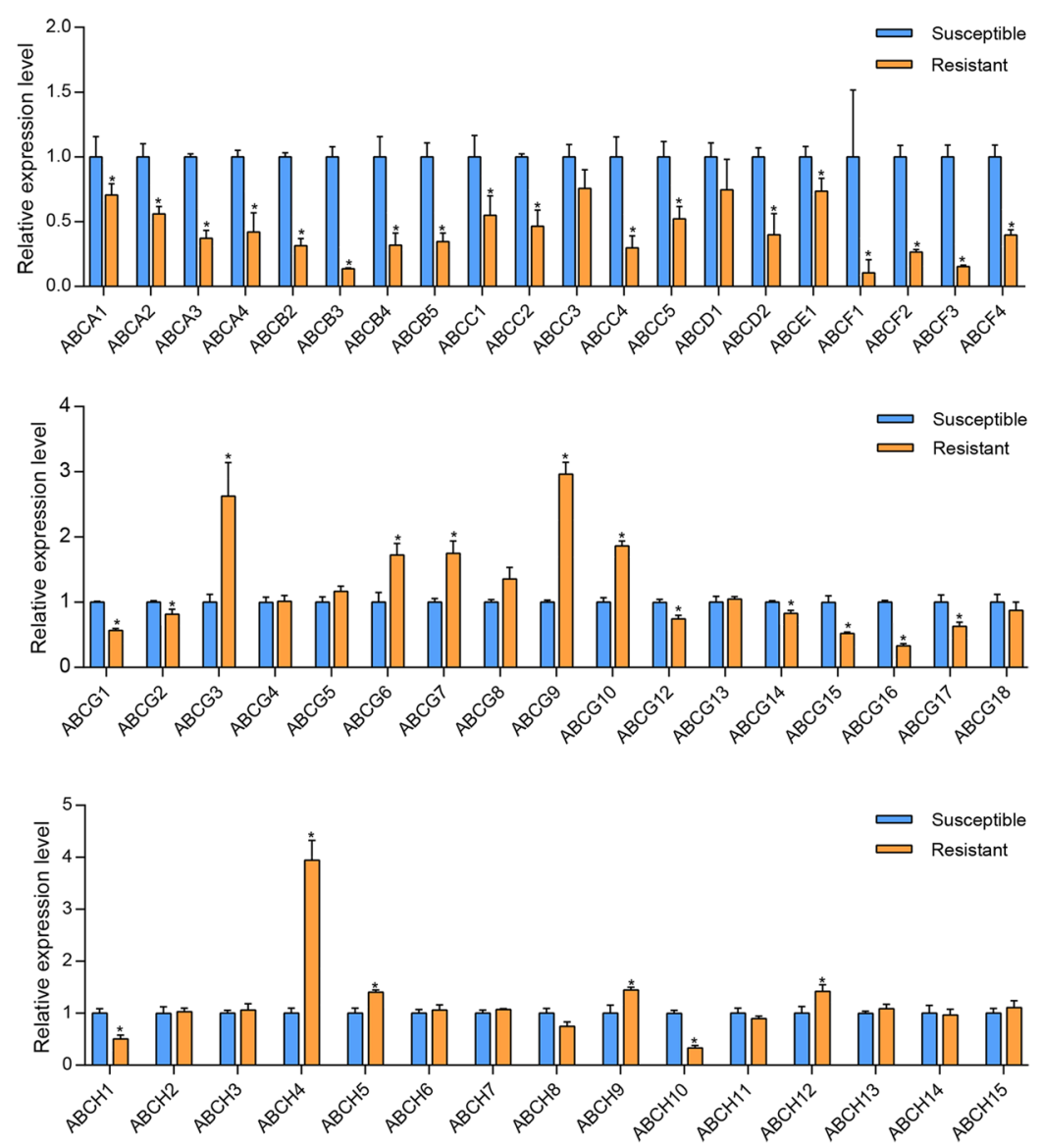

Figure 6. Transcript levels of 52 ATP-binding cassette (ABC) transporter genes in D. citri. The error bars indicate the standard errors of the means $(n=3)$, The asterisks indicate significant differences compared with the susceptible strain (Student's t-test, $\mathrm{P}<0.05$ ).

Identification of $A B C$ transporters in D. citri. The local blast program of BioEdit software was applied to identify candidate $\mathrm{ABC}$ transporter genes. The amino acid sequences of H. sapiens ${ }^{2}$, D. melanogaster ${ }^{30},{\text { B. } \text { mor }^{47}}^{47}$, T. castaneum $^{29}$, B. tabaci ${ }^{64}$, P. xylostella ${ }^{51}, T$. urtica $e^{30}$, and Saccharomyces cerevisiae ${ }^{77}$ were used as BLAST queries with an E-value threshold of $10^{-5}$. To obtain the whole $\mathrm{ABC}$ transporter genes as far as possible, the same method was also used to identify ABC transporters in the genome of D. citri (Accession: GCF_000475195.1). The candidate $\mathrm{ABC}$ transporter genes were reconfirmed by BLASTx analysis with the non-redundant protein sequence (NR) of NCBI (http://www.ncbi.nlm.nih.gov/).

Protein structure and domain prediction. The open reading frames (ORFs) of the candidate $A B C$ transporter genes were predicted using the ORF finder (http://www.ncbi.nlm.nih.gov/). The ORFs of the genes in each subfamily were aligned using ClastalW to search alternative splicings to confirm the isoforms from the same gene. The NBD and TMD of each potential ABC transporter genes were verified by searching with the Pfam program (http://pfam.xfam.org/) (78 $^{7}$ and Conserved Domains (http://www.ncbi.nlm.nih.gov//) ${ }^{79}$. The domains were graphed with the illustrator program DOG 2.0.1 ${ }^{80}$. N-glycosylation sites and O-glycosylation sites were predicted using NetNGlyc 1.0 (Potential Score $>0.5$, Jury agreement: 9/9) (http://www.cbs.dtu.dk/services/NetNGlyc/) and O-glycosylation ${ }^{81}$ (G-score $>0.5$ ) (http://www.cbs.dtu.dk/services/NetOGlyc-3.1/) respectively.

Phylogenetic analysis. To classify the position of D. citri $\mathrm{ABCs}$ within $\mathrm{ABC}$ classes (A-G), the amino acid sequences of NBDs of D. citri $\mathrm{ABC}$ transporters were used to resolve their phylogenetic relationships. When a protein had two NBDs, the N-terminal NBD was used. To analyse the evolutionary placement of ABC transporters in D. citri, comparison analyses among each subfamily of $\mathrm{ABC}$ transporters from D. citri, D. melanogaster, $B$. mori, T. castaneum, B. tabaci, and T. urticae were conducted, and the full-length protein sequences were subjected to phylogenetic analyses (Supplementary data). Sequences were aligned by the ClustalW alignment algorithm ${ }^{82}$, and MEGA6 was used to construct the neighbor-joining trees with the Poisson model and 1,000 bootstrap replicates $^{83}$, the dendrograms were viewed in FigTree and edited in Adobe PhotoShop CS6.

Expression analysis of $A B C$ transporters. Gene expression levels for each tissue sample were estimated by RSEM (RNA-Seq by Expectation-Maximization) (v1.2.6) ${ }^{84}$. The spatial expression of these ABC transporter genes was estimated using the Fragments Per Kilobase of transcript per Million fragments (FPKM) method, 
which is based on the number of uniquely mapped reads ${ }^{85}$. The FPKM values of each gene were transformed into log2 (RPKM + 1) values, and GraphPad Prism 7.01 was used to generate and visualize the expression profile.

The relative expression of $\mathrm{ABC}$ transporter mRNAs in imidacloprid-susceptible and imidacloprid-resistant strains was determined using quantitative real-time PCR (qRT-PCR) with SYBR-green fluorescence. Total RNA was extracted from two strains using TRIzol Reagent (Invitrogen, Waltham, MA, USA). The PrimeScript ${ }^{\mathrm{TM}}$ RT reagent Kit with gDNA Eraser (Takara, Dalian, China) was used for cDNA synthesis, and two reference genes, namely, $\beta$-actin (XM_008473151) and $\alpha$-tubulin (DQ675550), were used as internal controls ${ }^{86,87}$. The gene-specific primers were designed by the Primer 3 program (http://elixir.ut.ee/Main/Services) (Supplementary Table S1). Gene-specific primers synthesis were completed by TsingKe Biotech Co., Ltd (TsingKe, Beijing, China). The CFX96 Real-Time PCR System (Bio-Rad, Hercules, CA, USA) and the Go Taq ${ }^{\circledR}$ qPCR Master Mix (Promega, Madison, WI, USA) were used to perform qRT-PCR reactions. Finally, the relative values of mRNA expression were calculated by The $2^{-\Delta \Delta C t}$ method $^{88}$, and the expression level of imidacloprid-susceptible strain was used as the calibrator. The significance of differences between two strains was determined using Student's $t$ test $(\mathrm{P}<0.05)$. Three biological replicates were analysed for each experiment. A total of 120 ACP adults (three biological replicates, $\mathrm{n}=40$ ) from susceptible and resistant strains were used for qRT-PCR analysis, and three technical replicates were performed in each qRT-PCR reaction.

Received: 14 January 2019; Accepted: 15 October 2019;

Published online: 01 November 2019

\section{References}

1. Higgins, C. F. ABC transporters: from microorganisms to man. Annu. Rev. Cell Biol. 8, 67-113, https://doi.org/10.1146/annurev. cb.08.110192.0004358 (1992).

2. Dean, M., Rzhetsky, A. \& Allikmets, R. The human ATP-binding cassette (ABC) transporter superfamily. Genome Res. 11, 1156-1166, https://doi.org/10.1101/gr.GR-1649R (2001).

3. Dassa, E. \& Bouige, P. The ABC of ABCs: a phylogenetic and functional classification of ABC systems in living organisms. Res. Microbiol. 152, 211-229, https://doi.org/10.1016/S0923-2508(01)01194-9 (2001).

4. Rees, D. C., Johnson, E. \& Lewinson, O. ABC transporters: the power to change. Nat. Rev. Mol. Cell Bio. 10, 218-227, https://doi. org/10.1038/nrm2646 (2009)

5. Hollenstein, K., Dawson, R. J. \& Locher, K. P. Structure and mechanism of ABC transporter proteins. Curr. Opin. Struct. Biol. 17, 412-418, https://doi.org/10.1016/j.sbi.2007.07.003 (2007).

6. Bugde, P. et al. The therapeutic potential of targeting ABC transporters to combat multi-drug resistance. Expert Opin. Ther. Targets 21, 511-530, https://doi.org/10.1080/14728222.2017.1310841 (2017).

7. Chen, Z. et al. Mammalian drug efflux transporters of the ATP binding cassette (ABC) family in multidrug resistance: A review of the past decade. Cancer Lett. 370, 153-164, https://doi.org/10.1016/j.canlet.2015.10.010 (2016).

8. Lespine, A., Ménez, C., Bourguinat, C. \& Prichard, R. K. P-glycoproteins and other multidrug resistance transporters in the pharmacology of anthelmintics: Prospects for reversing transport-dependent anthelmintic resistance. Int. J. Parasitol. Drugs Drug Resist. 2, 58-75, https://doi.org/10.1016/j.ijpddr.2011.10.001 (2012).

9. Ardelli, B. F. Transport proteins of the ABC systems superfamily and their role in drug action and resistance in nematodes. Parasitol. Int. 62, 639-646, https://doi.org/10.1016/j.parint.2013.02.008 (2013).

10. You, M. et al. A heterozygous moth genome provides insights into herbivory and detoxification. Nat. Genet. 45, 220-225, https://doi. org/10.1038/ng.2524 (2013).

11. Xia, J. et al. Proteomics-based identification of midgut proteins correlated with Cryl Ac resistance in Plutella xylostella (L.). Pestic. Biochem. Physiol. 132, 108-117, https://doi.org/10.1016/j.pestbp.2016.01.002 (2016).

12. Sun, H., Pu, J., Chen, F., Wang, J. \& Han, Z. Multiple ATP-binding cassette transporters are involved in insecticide resistance in the small brown planthopper, Laodelphax striatellus. Insect Mol. Biol. 26, 343-355, https://doi.org/10.1111/imb.12299 (2017).

13. Gellatly, K. J. et al. RNAi validation of resistance genes and their interactions in the highly DDT-resistant 91-R strain of Drosophila melanogaster. Pestic. Biochem. Physiol. 121, 107-115, https://doi.org/10.1016/j.pestbp.2015.01.001 (2015).

14. Porretta, D. et al. How heterogeneous is the involvement of ABC transporters against insecticides? Acta Trop. 157, 131-135, https:// doi.org/10.1016/j.actatropica.2016.02.002 (2016).

15. Dermauw, W. \& Van Leeuwen, $\mathrm{T}$. The ABC gene family in arthropods: Comparative genomics and role in insecticide transport and resistance. Insect Biochem. Mol. Biol. 45, 89-110, https://doi.org/10.1016/j.ibmb.2013.11.001 (2014).

16. Buss, D. S. \& Callaghan, A. Interaction of pesticides with p-glycoprotein and other ABC proteins: A survey of the possible importance to insecticide, herbicide and fungicide resistance. Pestic. Biochem. Physiol. 90, 141-153, https://doi.org/10.1016/j. pestbp.2007.12.001 (2008).

17. Tiwari, S., Clayson, P. J., Kuhns, E. H. \& Stelinski, L. L. Effects of buprofezin and diflubenzuron on various developmental stages of Asian citrus psyllid, Diaphorina citri. Pest Manag. Sci. 68, 1405-1412, https://doi.org/10.1002/ps.3323 (2012).

18. Tiwari, S., Mann, R. S., Rogers, M. E. \& Stelinski, L. L. Insecticide resistance in field populations of Asian citrus psyllid in Florida. Pest Manag. Sci. 67, 1258-1268, https://doi.org/10.1002/ps.2181 (2011).

19. Naeem, A., Freed, S., Jin, F. L., Akmal, M. \& Mehmood, M. Monitoring of insecticide resistance in Diaphorina citri Kuwayama (Hemiptera: Psyllidae) from citrus groves of Punjab, Pakistan. Crop Prot. 86, 62-68, https://doi.org/10.1016/j.cropro.2016.04.010 (2016).

20. Kanga, L. H. B., Eason, J., Haseeb, M., Qureshi, J. \& Stansly, P. Monitoring for insecticide resistance in Asian Citrus Psyllid (Hemiptera: Psyllidae) populations in Florida. J. Econ. Entomol. 109, 832-836 (2016).

21. Qureshi, J. A., Kostyk, B. C. \& Stansly, P. A. Insecticidal suppression of Asian citrus psyllid Diaphorina citri (Hemiptera: Liviidae) vector of Huanglongbing pathogens. PLOS ONE 9, e112331, https://doi.org/10.1371/journal.pone.0112331 (2014).

22. Tiwari, S., Killiny, N. \& Stelinski, L. L. Dynamic insecticide susceptibility changes in Florida populations of Diaphorina citri (Hemiptera: Psyllidae). J. Econ. Entomol. 106, 393-399, https://doi.org/10.1093/jee/tov348 (2013).

23. Barr, N. B. et al. Comparison of laboratory colonies and field populations of Tamarixia radiata, an ectoparasitoid of the Asian citrus psyllid, using internal transcribed spacer and cytochrome oxidase subunit I DNA sequences. J. Econ. Entomol. 102, 2325-2332, https://doi.org/10.1603/029.102.0639 (2009).

24. Tiwari, S., Stelinski, L. L. \& Rogers, M. E. Biochemical basis of organophosphate and carbamate resistance in Asian citrus psyllid. J. Econ. Entomol. 105, 540-548, https://doi.org/10.1603/EC11228 (2012).

25. Tian, F., Li, C., Wang, Z., Liu, J. \& Zeng, X. Identification of detoxification genes in imidacloprid-resistant Asian Citrus Psyllid (Hemiptera: Lividae) and their expression patterns under stress of eight insecticides. Pest Manag. Sci. 75, 1400-1410, https://doi. org/10.1002/ps.5260 (2019). 
26. Hull, J. J. et al. Transcriptome-based identification of ABC transporters in the western tarnished plant bug Lygus hesperus. PLoS ONE 9, el13046, https://doi.org/10.1371/journal.pone.0113046 (2014).

27. Vaughan, A. M. \& Oram, J. F. ABCA1 and ABCG1 or ABCG4 act sequentially to remove cellular cholesterol and generate cholesterol-rich HDL. J. Lipid Res. 47, 2433-2443, https://doi.org/10.1194/jlr.M600218-JLR200 (2006).

28. Akram, A., Schmeidler, J., Katsel, P., Hof, P. R. \& Haroutunian, V. Increased expression of cholesterol transporter ABCA1 is highly correlated with severity of dementia in AD hippocampus. Brain Res. 1318, 167-177, https://doi.org/10.1016/j.brainres.2010.01.006 (2010).

29. Broehan, G., Kroeger, T., Lorenzen, M. \& Merzendorfer, H. Functional analysis of the ATP-binding cassette (ABC) transporter gene family of Tribolium castaneum. BMC Genomics 14, 6, https://doi.org/10.1186/1471-2164-14-6 (2013).

30. Dermauw, W. et al. A burst of ABC genes in the genome of the polyphagous spider mite Tetranychus urticae. BMC Genomics 14, 317, https://doi.org/10.1186/1471-2164-14-317 (2013).

31. Paterson, J. K. et al. Human ABCB6 localizes to both the outer mitochondrial membrane and the plasma membrane. Biochemistry 46, 9443-9452, https://doi.org/10.1021/bi700015m (2007).

32. Nikpour, M. et al. Papaemmanuil, E.; Högstrand, K. et al. The transporter ABCB7 is a mediator of the phenotype of acquired refractory anemia with ring sideroblasts. Leukemia 27, 889-896, https://doi.org/10.1038/leu.2012.298 (2012).

33. Elliott, A. M. \& Al-Haji, M. A. ABCB8 mediates doxorubicin resistance in melanoma cells by protecting the mitochondrial genome. Mol. Cancer Res. 7, 79-87, https://doi.org/10.1158/1541-7786.MCR-08-0235 (2009).

34. Liesa, M., Qiu, W. \& Shirihai, O. S. Mitochondrial ABC transporters function: the role of ABCB10 (ABC-me) as a novel player in cellular handling of reactive oxygen species. Biochim. Biophys. Acta Mol. Cell Res. 1823, 1945-1957, https://doi.org/10.1016/j. bbamcr.2012.07.013 (2012).

35. Sooksa-nguan, T. et al. Drosophila ABC transporter, DmHMT-1, confers tolerance to cadmium. J. Biol. Chem. 284, 354-362, https:// doi.org/10.1074/jbc.M806501200 (2008)

36. Ricardo, S. \& Lehmann, R. An ABC transporter controls export of a Drosophila germ cell attractant. Science. 323, 943-946, https:// doi.org/10.1126/science.1166239 (2009)

37. Bariami, V., Jones, C. M., Poupardin, R., Vontas, J. \& Ranson, H. Gene amplification, ABC transporters and cytochrome P450s: unraveling the molecular basis of pyrethroid resistance in the Dengue vector, Aedes aegypti. PLoS Negl. Trop. Dis. 6, e1692, https:// doi.org/10.1371/journal.pntd.0001692 (2012).

38. Moreau, C., Prost, A., Dérand, R. \& Vivaudou, M. SUR, ABC proteins targeted by KATP channel openers. J. Mol. Cell. Cardiol. 38, 951-963, https://doi.org/10.1016/j.yjmcc.2004.11.030 (2005).

39. Sheppard, D. N. \& Welsh, M. J. Structure and function of the CFTR chloride channel. Physiol. Rev. 79, S23-S45, https://doi. org/10.1152/physrev.1999.79.1.S23 (1999).

40. Chahine, S. \& Donnell, O. M. J. Physiological and molecular characterization of methotrexate transport by malpighian tubules of adult Drosophila melanogaster. J. Insect Physiol. 55, 927-935, https://doi.org/10.1016/j.jinsphys.2009.06.005 (2009).

41. Deeley, R. G., Westlake, C. \& Cole, S. P. C. Transmembrane transport of endo- and xenobiotics by mammalian ATP-Binding cassette multidrug resistance proteins. Physiol. Rev. 86, 849-899, https://doi.org/10.1152/physrev.00035.2005 (2006).

42. Bao, Y. et al. Triazophos up-regulated gene expression in the female brown planthopper, Nilaparvata lugens. J. Insect Physiol. 56, 1087-1094, https://doi.org/10.1016/j.jinsphys.2010.03.004 (2010).

43. Yoon, K. S. et al. Brief exposures of human body lice to sublethal amounts of ivermectin over-transcribes detoxification genes involved in tolerance. Insect Mol. Biol. 20, 687-699, https://doi.org/10.1111/j.1365-2583.2011.01097.x (2011).

44. Kruh, G. D., Guo, Y., Hopper-Borge, E., Belinsky, M. G. \& Chen, Z. ABCC10, ABCC11, and ABCC12. Pflugers Arch. 453, 675-684, https://doi.org/10.1007/s00424-006-0114-1 (2007).

45. Hopper-Borge, E. et al. Human multidrug resistance protein 7 (ABCC10) is a resistance factor for nucleoside analogues and Epothilone B. Cancer Res. 69, 178-184, https://doi.org/10.1158/0008-5472.CAN-08-1420 (2009).

46. Theodoulou, F. L., Holdsworth, M. \& Baker, A. Peroxisomal ABC transporters. FEBS Lett. 580, 1139-1155, https://doi.org/10.1042/ BST20150127 (2006).

47. Liu, S. et al. Genome-wide identification and characterization of ATP-binding cassette transporters in the silkworm, Bombyx mori. BMC Genomics 12, 491, https://doi.org/10.1186/1471-2164-12-491 (2011).

48. Zhao, Z., Fang, L. L., Johnsen, R. \& Baillie, D. L. ATP-binding cassette protein E is involved in gene transcription and translation in Caenorhabditis elegans. Biochem. Biophys. Res. Commun. 323, 104-111, https://doi.org/10.1016/j.bbrc.2004.08.068 (2004).

49. Smirnova, E. V. et al. TULA proteins bind to ABCE-1, a host factor of HIV-1 assembly, and inhibit HIV-1 biogenesis in a UBAdependent fashion. Virology 372, 10-23, https://doi.org/10.1016/j.virol.2007.10.012 (2008).

50. Paytubi, S. et al. ABC50 promotes translation initiation in mammalian cells. J. Biol. Chem. 284, 24061-24073, https://doi. org/10.1074/jbc.M109.031625 (2009).

51. Qi, W. et al. Characterization and expression profiling of ATP-binding cassette transporter genes in the diamondback moth, Plutella xylostella (L.). BMC Genomics 17, 760, https://doi.org/10.1186/s12864-016-3096-1 (2016).

52. Kovalchuk, A. \& Driessen, A. J. Phylogenetic analysis of fungal ABC transporters. BMC Genomics 11, 177, https://doi. org/10.1186/1471-2164-11-177 (2010).

53. Verrier, P. J. et al. Plant ABC proteins - a unified nomenclature and updated inventory. Trends Plant Sci. 13, 151-159, https://doi. org/10.1016/j.tplants.2008.02.001 (2008).

54. Fukuda, Y. \& Schuetz, J. D. ABC transporters and their role in nucleoside and nucleotide drug resistance. Biochem. Pharmacol. 83, 1073-1083, https://doi.org/10.1016/j.bcp.2011.12.042 (2012).

55. Sullivan, D. T., Grillo, S. L. \& Kitos, R. J. Subcellular localization of the first three enzymes of the ommochrome synthetic pathway in Drosophila melanogaster. J. Exp. Zool. 188, 225-233, https://doi.org/10.1002/jez.1401880210 (1974).

56. Kerr, I. D., Haider, A. J. \& Gelissen, I. C. The ABCG family of membrane-associated transporters: you don't have to be big to be mighty. Br. J. Pharmacol. 164, 1767-1779, https://doi.org/10.1111/j.1476-5381.2010.01177.x (2011).

57. Ewart, G. D. \& Howells, A. J. ABC transporters involved in transport of eye pigment precursors in Drosophila melanogaster. Meth. Enzymol. 292, 213-224, https://doi.org/10.1016/S0076-6879(98)92017-1 (1998)

58. Mackenzie, S. M. et al. Mutations in the white gene of Drosophila melanogaster affecting ABC transporters that determine eye colouration. Biochim. Biophys. Acta Biomembr. 1419, 173-185, https://doi.org/10.1016/S0005-2736(99)00064-4 (1999).

59. Kômoto, N., Quan, G., Sezutsu, H. \& Tamura, T. A single-base deletion in an ABC transporter gene causes white eyes, white eggs, and translucent larval skin in the silkworm w-3oe mutant. Insect Biochem. Mol. Biol. 39, 152-156, https://doi.org/10.1016/j. ibmb.2008.10.003 (2009).

60. Guo, Z. et al. Down-regulation of a novel ABC transporter gene (Pxwhite) is associated with Cryl Ac resistance in the diamondback moth, Plutella xylostella (L.). Insect Biochem. Mol. Biol. 59, 30-40, https://doi.org/10.1016/j.ibmb.2015.01.009 (2015).

61. Hock, T., Cottrill, T., Keegan, J. \& Garza, D. The E23 early gene of Drosophila encodes an ecdysone-inducible ATP-binding cassette transporter capable of repressing ecdysone-mediated gene activation. Proc. Natl. Acad. Sci. USA 97, 9519-9524, https://doi. org/10.1073/pnas.160271797 (2000).

62. Mummery-Widmer, J. L. et al. Genome-wide analysis of notch signalling in Drosophila by Transgenic RNAi. Nature. 458, 987-992, https://doi.org/10.1038/nature07936 (2009). 
63. Jeong, C. et al. Genome-wide identification of ATP-binding cassette (ABC) transporters and conservation of their xenobiotic transporter function in the monogonont rotifer (Brachionus koreanus). Comp. Biochem. Physiol. Part D Genomics Proteomics 21, 17-26, https://doi.org/10.1016/j.cbd.2016.10.003 (2017).

64. Tian, L. et al. Genome-wide analysis of ATP-binding cassette (ABC) transporters in the sweetpotato whitefly, Bemisia tabaci. BMC Genomics 18, 330, https://doi.org/10.1186/s12864-017-3706-6 (2017).

65. Sturm, A., Cunningham, P. \& Dean, M. The ABC transporter gene family of Daphnia Pulex. BMC Genomics 10, 170, https://doi. org/10.1186/1471-2164-10-170 (2009).

66. Koenig, C. et al. The plastic response of Manduca sexta to host and non-host plants. Insect Biochem. Mol. Biol. 63, 72-85, https://doi. org/10.1016/j.ibmb.2015.06.001 (2015).

67. Bretschneider, A., Heckel, D. G. \& Vogel, H. Know your ABCs: Characterization and gene expression dynamics of ABC transporters in the polyphagous herbivore Helicoverpa armigera. Insect Biochem. Mol. Biol. 72, 1-9, https://doi.org/10.1016/j.ibmb.2016.03.001 (2016).

68. Qin, W., Neal, S. J., Robertson, R. M., Westwood, J. T. \& Walker, V. K. Cold hardening and transcriptional change in Drosophila melanogaster. Insect Mol. Biol. 14, 607-613, https://doi.org/10.1111/j.1365-2583.2005.00589.x (2005).

69. Zhang, S., Feany, M. B., Saraswati, S., Littleton, J. T. \& Perrimon, N. Inactivation of Drosophila Huntingtin affects long-term adult functioning and the pathogenesis of a Huntington's disease model. Dis. Model. Mech. 2, 247-266, https://doi.org/10.1242/ dmm.000653 (2009).

70. Zuber, R. et al. The ABC transporter Snu and the extracellular protein Snsl cooperate in the formation of the lipid-based inward and outward barrier in the skin of Drosophila. Eur. J. Cell Biol. 97, 90-101, https://doi.org/10.1016/j.ejcb.2017.12.003 (2018).

71. Yu, Z. et al. The ABC transporter ABCH-9C is needed for cuticle barrier construction in. Locusta Migratoria. Insect Biochem. Mol. Biol. 87, 90-99, https://doi.org/10.1016/j.ibmb.2017.06.005 (2017).

72. Kaplanoglu, E., Chapman, P., Scott, I. M. \& Donly, C. Overexpression of a cytochrome P450 and a UDP-glycosyltransferase is associated with imidacloprid resistance in the Colorado potato beetle, Leptinotarsa decemlineata. Sci. Rep. 7, 1762, https://doi. org/10.1038/s41598-017-01961-4 (2017).

73. Silva, A. X., Jander, G., Samaniego, H., Ramsey, J. S. \& Figueroa, C. C. Insecticide resistance mechanisms in the green peach aphid Myzus persicae (Hemiptera: Aphididae) I: a transcriptomic survey. PLoS One 7, e36366, https://doi.org/10.1371/journal. pone.0036366 (2012)

74. Grabherr, M. G. et al. Full-length transcriptome assembly from RNA-Seq data without a reference genome. Nat. Biotechnol. 29, 644-652, https://doi.org/10.1038/nbt.1883 (2011)

75. Yin, C. et al. InsectBase: A resource for insect genomes and transcriptomes. Nucleic Acids Res. 44, D801-D807, https://doi. org/10.1093/nar/gkv1204 (2016).

76. Wang, Z., Zhou, W., Hameed, M., Liu, J. \& Zeng, X. Characterization and expression profiling of neuropeptides and G-ProteinCoupled Receptors (GPCRs) for neuropeptides in the Asian citrus psyllid, Diaphorina citri (Hemiptera: Psyllidae). Int. J. Mol. Sci. 19,3912, https://doi.org/10.3390/ijms19123912 (2018).

77. Taglicht, D. \& Michaelis, S. Saccharomyces cerevisiae ABC proteins and their relevance to human health and disease. Meth. Enzymol. 292, 130-162, https://doi.org/10.1016/S0076-6879(98)92012-2 (1998).

78. Finn, R. D. et al. The Pfam protein families database: towards a more sustainable future. Nucleic Acids Res. 44, D279-D285, https:// doi.org/10.1093/nar/gkv1344 (2016).

79. Marchler-Bauer, A. et al. CDD/SPARCLE: functional classification of proteins via subfamily domain architectures. Nucleic Acids Res. 45, D200-D203, https://doi.org/10.1093/nar/gkw1129(2017).

80. Ren, J. et al. DOG 1.0: illustrator of protein domain structures. Cell Res. 19, 271-273, https://doi.org/10.1038/cr.2009.6 (2009).

81. Julenius, K., Lgaard, M., Gupta, A. \& Brunak, R. S. R. Prediction, conservation analysis, and structural characterization of mammalian mucin-type O-glycosylation sites. Glycobiology 15, 153-164, https://doi.org/10.1093/glycob/cwh151 (2005).

82. Thompson, J. D., Higgins, D. G. \& Gibson, T. J. CLUSTAL W: improving the sensitivity of progressive multiple sequence alignment through sequence weighting, position-specific gap penalties and weight matrix choice. Nucleic Acids Res. 22, 4673-4680, https://doi. org/10.1093/nar/22.22.4673(1994).

83. Tamura, K., Stecher, G., Peterson, D., Filipski, A. \& Kumar, S. MEGA6: molecular evolutionary genetics analysis version 6.0. Mol. Biol. Evol. 30, 2725-2729, https://doi.org/10.1093/molbev/mst197 (2013).

84. Li, B. \& Dewey, C. N. RSEM: Accurate transcript quantification from RNA-Seq data with or without a reference genome. BMC Bioinformatics 12, 323, https://doi.org/10.1186/1471-2105-12-323 (2011).

85. Anders, S. \& Huber, W. Differential expression analysis for sequence count data. Genome Biol. 11, R106, https://doi.org/10.1186/ gb-2010-11-10-r106 (2010).

86. Tian, F., Wang, Z., Li, C., Liu, J. \& Zeng, X. UDP-glycosyltransferases are involved in Imidacloprid resistance in the Asian Citrus Psyllid, Diaphorina citri (Hemiptera: Lividae). Pestic. Biochem. Physiol. 154, 23-31, https://doi.org/10.1016/j.pestbp.2018.12.010 (2019).

87. Yu, X. \& Killiny, N. RNA interference of two glutathione S-transferase genes, Diaphorina citri DcGSTe2 and DcGSTd1, increases the susceptibility of Asian Citrus Psyllid (Hemiptera: Liviidae) to the pesticides fenpropathrin and thiamethoxam. Pest Manag. Sci. 74, 638-647, https://doi.org/10.1002/ps.4747 (2018).

88. Livak, K. J. \& Schmittgen, T. D. Analysis of relative gene expression data using real-time quantitative PCR and the $2^{-\Delta \Delta C T}$ method. Methods 25, 402-408, https://doi.org/10.1006/meth.2001.1262 (2001).

\section{Acknowledgements}

This research was funded by the National Natural Science Foundation of China, 31572314 and 31971424; National Key Research and Development Program of China, 2017YFD0202005; Doctoral Innovative Talents Training Project of South China Agricultural University, CX2019N047. The authors sincerely thank Dr. Zihua Wang for editorial assistance on the manuscript, and Miss Xiaoyue Sang for the construction of dendrograms.

\section{Author contributions}

Z.W., F.T. and X.Z. conceived and designed the experimental plan. Z.W. wrote the main manuscript text and conducted the experiments. Z.W. and F.T. performed the experiments. Z.W. analysed the results. L.C., J.Z. and J.L. provided some suggestions for revision and corrected the grammatical mistakes. X.Z. supervised the study. All authors reviewed the manuscript.

\section{Competing interests}

The authors declare no competing interests.

\section{Additional information}

Supplementary information is available for this paper at https://doi.org/10.1038/s41598-019-52402-3. 
Correspondence and requests for materials should be addressed to X.Z.

Reprints and permissions information is available at www.nature.com/reprints.

Publisher's note Springer Nature remains neutral with regard to jurisdictional claims in published maps and institutional affiliations.

(c) Open Access This article is licensed under a Creative Commons Attribution 4.0 International License, which permits use, sharing, adaptation, distribution and reproduction in any medium or format, as long as you give appropriate credit to the original author(s) and the source, provide a link to the Creative Commons license, and indicate if changes were made. The images or other third party material in this article are included in the article's Creative Commons license, unless indicated otherwise in a credit line to the material. If material is not included in the article's Creative Commons license and your intended use is not permitted by statutory regulation or exceeds the permitted use, you will need to obtain permission directly from the copyright holder. To view a copy of this license, visit http://creativecommons.org/licenses/by/4.0/.

(C) The Author(s) 2019 